\title{
Investigation of Adult Education Program Planning From a Power and Interest Perspective: A Case Study of a Transnational Ph.D. Cohort Program
}

\author{
Chi-Hu Tien \\ Hungkuang University, Taichung, Taiwan
}

\begin{abstract}
Program planning is the major channel currently utilized to achieve excellent standards in adult educational practice. Through well-planned programs, adult education policies can be implemented, organizational goals can be realized, learners' behaviors can be positively altered, and their abilities enhanced. It is worth emphasizing that traditional models of program planning applying linear paths do not explain a multitude of situations that occur in reality. This is largely due to the fact that linear models generally ignore the role and influence of political factors in the program planning processes. Centered on the perspective of power and interest negotiation in program planning and utilizing a case study approach, this study investigated and analyzed the program planning processes of a Ph.D. Cohort program in an American university in Taiwan. In addition to transforming the power and interest negotiation perspective into a diagram to interpret the case, four new discoveries were made. Firstly, interests should be further divided into tangible and intangible interests. Secondly, timing was found to be an additional factor that influences various stakeholders in the program planning process. Thirdly, stakeholders of "invisible" intangible interests may influence the program-planning process. Finally, the degree of power was determined to be the major impact factor in the program planning process.
\end{abstract}

Keywords: program planning, power, interest negotiation

\section{Introduction}

Program planning refers to the process of developing educational programs that achieve objectives and solve problems by stimulating extensive consideration, alongside the compilation and analysis of currently existing teaching resources and learning materials. The steps involved in educational program planning include administrative and organizational management related content, such as analyzing the context, generating the initial program design, defining target learners and program objectives, understanding learners' needs, designing learning activities, promoting the program, evaluating results, and applying budgetary control and process management practices. Program planning is the primary route in achieving excellence in adult educational practice. Through planned programming, adult educational policies are implemented, institutional goals realized, and learners' behaviors positively altered, and their abilities enhanced. Therefore, there are at least 100 models related to program development in the existing literature, with numerous books and journal articles extensively covering the subject (Wei, 2001). However, traditional linear-thinking program planning

Chi-Hu Tien, Ph.D., assistant professor, Department of Cultural and Creative Industries, Hungkuang University. 
UImodels lack the ability to accurately explain situations that occur in reality, and thus, are rarely applied to real life cases. Such examples include the political aspirations proposed by the Taiwanese government, such as "Balance the Development of Northern and Southern Taiwan," "Ensure Fairness and Justice," and "Love Taiwan."

\section{Politics in Program Planning}

Organizations are alliances composed of different individuals and interest groups. Since there are discrepancies in values, preferences, beliefs, and perceptions of reality between individuals and the group, conflicts become unavoidable. In Das Kapital (Capital), Marx referred to politics as the superstructure of human society, which determines the substructure (or base). In human society, politics is universal. It is impossible to avoid politics, as it exists in all forms of human activity. Boone, Safrit, and Jones (2002) pointed out that any study of power and influence inevitably tends to mention "politics." Politics is formed when individuals and groups intend to avoid head-on confrontation yet desire to reach a settlement. For that reason, Sissel's (2001) book begins with the sentence "It is all political."

In Planning Responsibly for Adult Education: A Guide to Negotiating Power and Interests, Cervero and Wilson (1994) put forward ideal standards for the study of program planning. In the first chapter of the book, the writers pointed out that program planning is a social activity, in which people negotiate the interests of both the individuals and the organization. Educational programs are planned by a group of real persons in a complex organization. This complex organization has historical traditions, power relations, as well as human needs and interests.

Since programs are planned by real people in a real social context, they are not likely to be similar to a controlled model constructed in a laboratory, nor are they likely to follow clearly defined steps with a linear logic design, based on rational thinking. The final design tends to be shaped based on the political judgment of the planner, such as his/her personal interests and power motives. For that reason, if we are unable to understand the political connotations and operations behind program planning, then we are unable to arrive at the practical aspects of program planning, thus making the theory and practice less likely to be compatible in a real-world setting. The power and interest negotiation aspects in program planning proposed by Cervero and Wilson (1994) had provided a persuasive perspective on the political processes involved, as well as their influence during the planning of educational programs.

This study utilized a Ph.D. Cohort program conducted by an American university in Taiwan as the subject of the case study. In addition to collecting relevant information on the program in Taiwan, open and in-depth interviews were conducted among six stakeholders for further information. The seven interviewees were Professor A (A professor from the American university), Teacher B (A teacher from a Taiwanese company that had contacted the university to introduce the program into the Taiwanese market), a person from the Taiwanese market that was responsible for the planning and implementation of the program, two students involved in the program, and the two alumni members of the university in Taiwan. In this study, Cervero and Wilson's (1994) theory of power and interest negotiation based program planning was first transformed into a graphic model; then, the constructed model was utilized to examine and analyze the planning phases in the selected case. Finally, suggestions were proposed accordingly.

\section{Power and Interest Negotiation in Program Planning}

\section{The Different Viewpoints}

Chien (2004) pointed out that when discussing the politics of power, Lakoff (1990) suggested that the 
power in an organization interacts and should be divided into microscopic and macroscopic forms of power. The microscopic manifestation of power refers to the behaviors of developing and utilizing strategies to create and enhance interpersonal differences in power and the effects of power. The macroscopic manifestation of power refers to the interaction between groups or organizations. As opposed to the traditional theories of macro-politics, micro-politics takes the perspective of political behavior and emphasizes the interaction processes between the characteristics of organizational context and individual members. Therefore, when studying the dynamics of power in an organization, it is suggested to approach from the most subtle and detailed components of the organization in order to examine the historical context, development trajectories, and strategies. Also, to explore the influential factors of politics in the organization, such as power, interests, conflicts, the potential mentality, and attitude of individual members during the interaction process. Utilization of power strategies resolves conflicts of interests, decision-making, negotiation, and formation of allies.

It is believed that people jointly participate in the same task when their own interests can be realized. When conflicts occur due to the differentiated preferences of individuals and groups on a certain subject, negotiation is a process where all relevant parties can make a joint decision. More importantly, the objectives and acceptable proposals of the organization are established by the individual that possesses power during the negotiation process. Power is an important determinant in negotiation. It is seen as the ability to match the results of the negotiation to the expected outcome through desired means (Wei, 2001). According to Cervero and Wilson (1996), previous studies on program planning can be summarized as the following three viewpoints:

1. Classical viewpoint: The classical viewpoint focuses mainly on the development of a set of standard planning procedures for practitioners to follow. Boyle (1981) claimed in "Planning Better Programs" that program planning is the art of designing and implementing a course of actions in order to achieve effectiveness of educational programs. The classical viewpoint has long dominated the planning process of adult education. However, the effectiveness and practicality of standard and step-by-step operating procedures remain questionable in reality.

2. Naturalist viewpoint: The naturalist viewpoint believes that planners should not merely apply one set of standard operating principles and procedures to any particular context. Contrarily, each circumstance should be considered and decisions should be made accordingly. The naturalist viewpoint emphasizes that the planner should have the experience to make decisions according to the circumstances and be able to provide appropriate reasoning for the adoption of the decision. However, predefined standards, corresponding skills, and theories should be provided to planners before suitable decisions and plans can be made. Therefore, the naturalist models of program planning focus on the ethics and values during planning, as well as the impact of the nature of human interactions on the planning process.

3. Critical viewpoint: The critical viewpoint argues that planners need to understand that the planning process is a series of political and ideological activities that are associated with social inequality, before appropriate plans can be developed. The core concept of the critical viewpoint lies in that planning is a social, political, and ethical activity, which involves both deliberation and decision-making. In addition, the deliberation and decision-making process is related to an organization's function, history, interactions between members, and the power relations with other organizations. As such, it involves conflicts between different parties with their own interests and desires. Therefore, planners should pay attention to the relationship between conflicting parties and be sensitive to the timing of decisions made, as well as organizational structure. 
Cervero and Wilson (1998) pointed out that the classical viewpoint is an abstract and ideal system-planning model. However, it does not provide sufficient help to educators to understand the daily tasks involved. The naturalist viewpoint considers planners as practitioners who are required to be aware of the actual tasks involved in daily operations and argues that a useful planning theory should illustrate the actual tasks of the planner. The naturalist viewpoint emphasizes the importance of practical actions and understanding of the context where decisions and judgments need to be made. The critical viewpoint suggests that planners should understand that education is a political and ideological activity, which requires clear ethical standards, so as to play a more active role in the promotion of an increasingly progressive and equitable society. Therefore, Cervero and Wilson (2006) advocated that the first step in improving program planning is to transfer the plan from the planner's personal conception to the organizational context and treat the planning process as a social activity, in which people negotiate with one another to answer questions and find solutions to the problems related to the plans. Their interest negotiation model is a combination of complex assumptions, goals, values, desires, expectations, and other orientations that guide the direction of the planners' behavior. They argued that power should not be seen as a special dictatorial position, but rather the ability to take action. Power is generated from normal social relations, which are neither given nor can they be taken away, rather they are obtained through the process of negotiation. In their view, program planning is a form of political behavior. Program planners do not follow scripts and they are real people who are required to negotiate the interests of everyone within the existing power relations network, so that an educational program can be developed accordingly. In addition, planners require guidance from a responsible vision, and therefore, the retention of ethics is of great importance to the program planning process. If ethics is not retained, the outcome of any adult education program developed is likely to merely reflect the interests of the individuals with the greatest power.

\section{The Social Context Matters}

Power is an important determinant in negotiation and powers in negotiation can be further categorized into informational power, reward power, coercive power, legitimate power, expert power, and referent power. The possession of power means possessing the ability to influence the outcome of the negotiation, so that it matches the corresponding party's expectations. Only by negotiating with influential power can the outcome of the negotiation be guided towards a side that is beneficial to the corresponding party. The social contexts constructed by power and interests are where program planners are required to execute the tasks. Hence, the planners' actions should be associated with such contexts. On that account, negotiation could be said to be the main activity in the program planning phase. Negotiation usually involves the following two stages: The first stage corresponds to the traditional concept of negotiation, where planners are required to negotiate their own interests with the other party, as well as balance the interests of other parties with their own. The second stage mainly involves planners' negotiating with their own interests and power relations. This paradigm exists because program planning is a dynamic process, and during negotiation, planners may need to change plans, which may impact the planners' original interests and power relations, as well as generate new interests and power relations. Based on the aforementioned studies, the present study summarized seven key points in the power-and-interests program planning model:

1. Program planning is a social activity, during which individuals negotiate the interests of both individual members and organizations. In other words, interests can be divided into individual and organizational interests; 
2. Organizations have defined historical traditions, power relations, set needs of members, preferences, and interests;

3. It is the planner's responsibility to negotiate with all stakeholders of the program. Therefore, the negotiation phases should include all stakeholders;

4. The process of program planning is a dynamic process of negotiation, distribution, and re-distribution of interests;

5. Interests are a combination of complex assumptions, goals, values, desires, expectations, and other orientations;

6. Power relations exist in the interaction between all parties and determine the actions taken by the planners in any given context;

7. Power relations are constructed by the organizational and social context.

\section{Models of Power and Interest Negotiation}

Using "A," "B," and "C" to represent different individuals, and "O" to symbolize a given organization (for example, "AO" signifies the organization that "A" is a member of), the present researcher developed two graphic models to illustrate the impact of interests on individuals and organizations (see Figure 1) and the program planner's power relations and negotiation of interests (see Figure 2).

Cervero and Wilson (2006) repeatedly stressed in their book that program planners are real people that live in dynamic social contexts. Hence, they are influenced by various interests and the surrounding power relations. Based on the present author's practical experience in program planning, this argument is persuasive, as it does indeed reflect the situation in reality. Rational and linear models serve as an excellent reference. However, it is very rare that such models may be implemented in absolute accordance with the plan. This dissonance occurs less because the planners are reluctant to abide by the rules, and more that the idealistic plans are impractical in reality. Relevant research has elaborated the mechanisms of interests and power, as well as its relationship within the social and cultural context, and suggested that planners should be guided by ethics, so as to maintain appropriate beliefs and a clear direction, rather than be reduced to an instrument in the game of power and interests. However, few studies have specified the actual application of their statements and proposals in a real situation. In addition, questions surrounding the completeness of the influential factors on interest negotiation summarized by past studies, as well as how to define and observe power relations and their influence in the dynamic program planning process, remain unexplored.

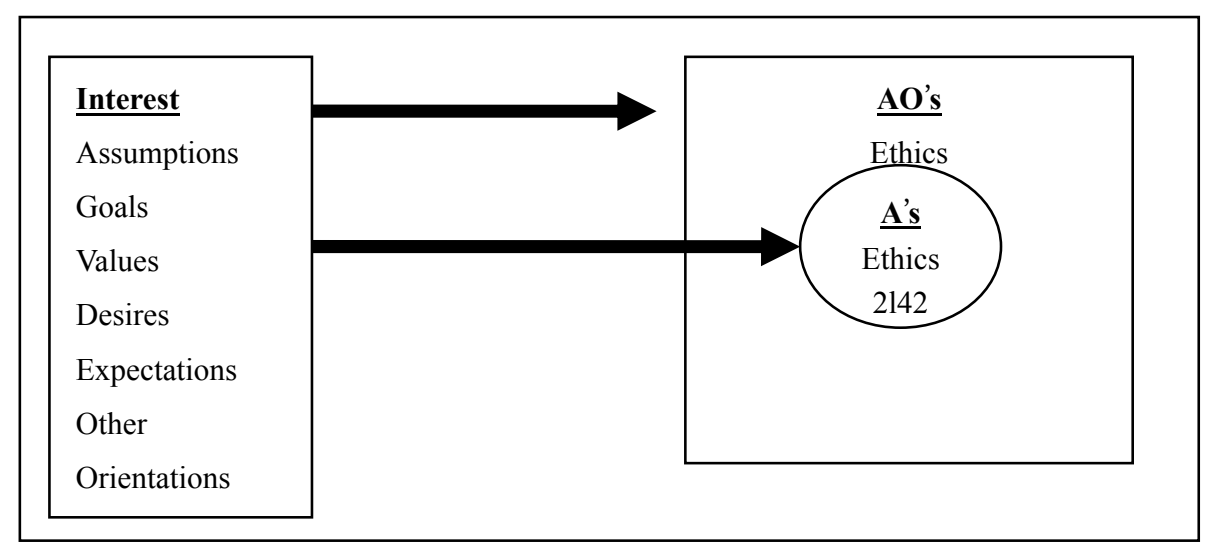

Figure 1. Graphic model of the impact of interests on individuals and organizations. 


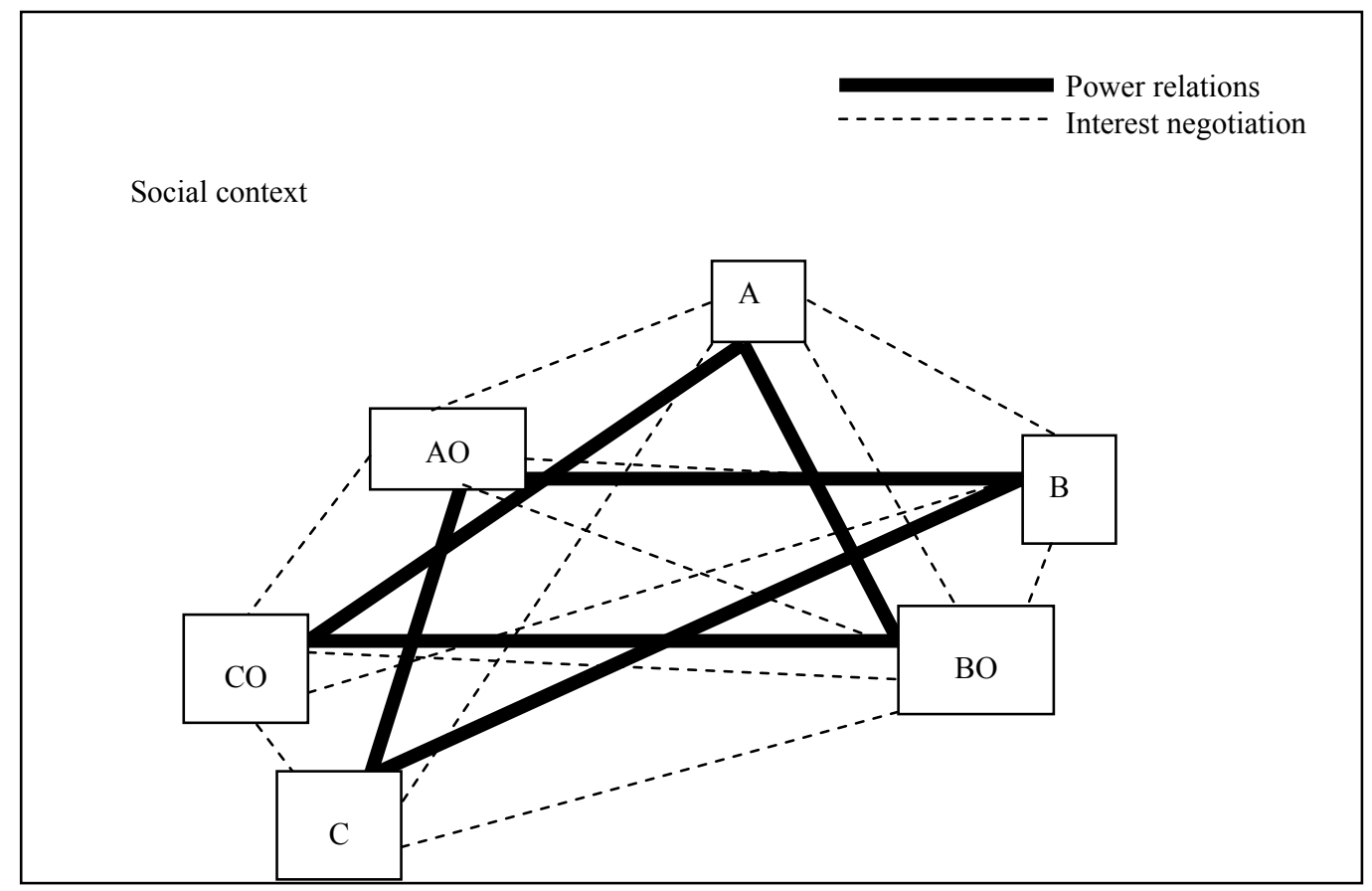

Figure 2. Graphic model of the planner's owner relations and interest negotiation.

\section{Results and Findings}

A Ph.D. Cohort program held by an American university in Taiwan (the program) was selected as the subject of a case study. In addition to collecting program-related information, open and in-depth interviews were conducted among the stakeholders of the program. The results showed that three projects were successfully analyzed from a power and interest negotiation perspective, using the proposed graphic models.

1. The influence of personal values is significant. In the case study, the investigated individual in charge of the planning and implementation of the program in Taiwan (the program planner) has remained a follower of culture and education since he was younger, and had always aspired towards a career in the field of culture and education in Taiwan. He had previously studied in the United States (U.S.) and thought highly of the American education system. Therefore, when he returned from the U.S. back to Taiwan, he began actively seeking a culture and education related job opportunity. When he was provided with the opportunity to work on the program, he was actively involved in the promotion and implementation of the program, even though he did not apply a sophisticated calculation of benefit and loss. With his devotion and effort, the program was implemented and had gained its first group of students. However, the American university gave no assurances to continue the program thereafter. Although the future remained uncertain, the program planner continued to devote and invest his time and effort in the program. The current researcher believes that is was in fact the program planner's desire for success that was the main driving force behind the successful implementation of the program. Since the program planner had a strong desire for success, he was willing to invest in the program regardless of the financial and labor costs. The program was thus successfully implemented. For example, in addition to hiring three people to assist in corresponding tasks, the initial investment in promoting the program cost more than New Taiwan (NT) three million dollars, whilst the total annual income of the program was only about NT nine million dollars. It can be seen that the influential factors in the case of the program planner 
involved goals, values, and expectations. It is worth noting, however, that the planner himself was not very clear about the reasons behind making such a decision.

2. The relationships between stakeholders are complex and non-equidistant. The promotion of the program encompassed two visible organizations, namely, the company that the program planner was working for (the Taiwanese company) and the American university, as well as stakeholders with other interests. The individual stakeholders that represented various interests included Professor A, the program planner, Teacher B (who was assisting communication with the American university and the Taiwanese company, who thereafter joined the Taiwanese company in reference). A Taiwanese alumnus of the American university who caused repeated intervention during the promotion and implementation processes of the program joined, too. Thus, it can be seen that the complexity of the planning process was staggered, with complex relationships between all relevant stakeholders. The changes in the power relations during the planning and promotion of the program echoed Cervero and Wilson's (2006) description of the dynamic changes and interactive effects of power in real social contexts. According to the findings of this study, the original department head of the American university was quite hesitant towards the program. Although he gave verbal consent for the execution of the program, he did not sign the final agreement, resulting in tensions in the power dynamics between Professor A and the department head. Nevertheless, a few months later, the head of the department and the corresponding person in charge of the program was changed due to the rotation of personnel in the university. Hence, Professor A was able to establish new communication and power relations with the new department head, and thereby, accelerate the application process of the program.

3. Personal and organizational interests need to be negotiated during each planning process. The conflicts, coordination, and integration of personal and organizational interests can be demonstrated by the role of Professor A. In addition to considering the interests of the American university such that the university would be willing to issue a license for the program, Professor A also needed to consider his own benefits obtained from the Taiwanese company. For that reason, he was required to balance the relevant interests of both parties in order to facilitate a successful implementation of the program.

Furthermore, the study also discovered that the following factors could not be fully explained using the power-and-interest negotiation approach.

1. Interests should be further divided into material and psychological interests. Money, reputation, and vanity are all interests. If interests are further categorized into internal interests (psychological interests) and external interests (material interests), then the reasons behind the continued promotion of the program regardless of financial returns can be further explored. If all influencing interests are uncovered, the model possesses more explanatory power.

2. Timing is a significant influential factor. One good example of the importance of good timing was that Professor A took advantage of the personnel turnover of the school to accelerate the implementation of the program. Timing should be considered as individual variable that existed independently. Under the influence of timing, all other variables become contingencies that can be changed. Therefore, timing is a critical factor in the program planning process. Cervero and Wilson (1998) also put forward the importance of change and reminded planners the importance of being sensitive towards timing and organizational structure. However, the present author believes that timing should be considered an important independent variable in future research.

3. The presence of "invisible" stakeholders should not be ignored. Past studies did not specify the role of indirect stakeholders. However, from this case, it was observed that the laws and regulations of the Taiwanese 
government acted as "invisible" stakeholders. In order to avoid restrictions stipulated by corresponding rules and regulations, the Taiwanese company chose to move the location of where the courses will be taught to Hong Kong. The alumni association of the American university was also an "invisible" stakeholder. They utilized informal channels to express their reluctance and rejection of the idea that the program was organized by such a minor company in Taiwan. Although the oppositions of the alumni were informal and indirect, they generated a certain degree of pressure in the promotion of the program.

4. The amount of power is the key. The case revealed that theoretical democratic negotiation is rarely seen during the planning process in reality. The development of the program uncovered apparently unequal power relations. In fact, Professor A held the dominant position during the negotiation processes. Democratic negotiation was not easily attained, as the inequality of power and distribution of power and resources directly determined the progress of the program. Therefore, the amount of power appears to be a key in determining the success of program planning.

With the findings above, this study proposed a new improved model of power and interest negotiation in program planning as shown in Figure 3.

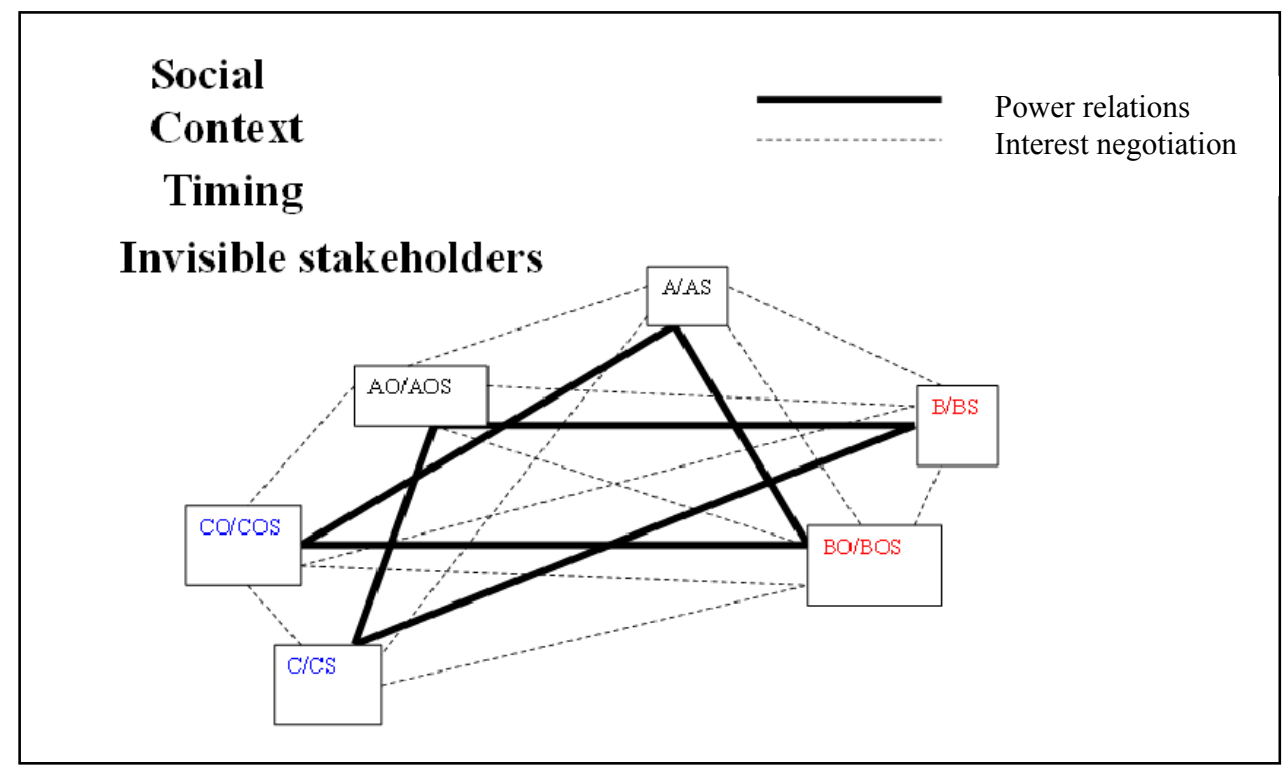

Figure 3. Improved Graphic Model of Planner'S Power Relations and interest negotiation.

\section{Conclusions}

Program planning is a holistic process that involves analysis and thinking. In addition to being familiar with the objectives of the program and learning content, program planners are faced with the complexities of human interactions during the planning process. Program planners are thus required to play the role of analyst, activist, facilitator, and stimulator, while retaining a certain degree of flexibility when promoting and implementing the program, and thereby negotiating an optimal solution for the network of power and interests. On that account, understanding the different interests of each stakeholder in the planning process (regardless of being monetary benefits, goals, expectations, or ethics) affects the outcome of the program.

Cervero and Wilson provided a satisfactory theoretical basis and research paradigm for the study of program planning politics, and their viewpoints and models can also be used to explain cases in Taiwan. 
However, from the case adopted in this study, it can be seen that not all aspects of the adult educational practice in Taiwan could be explained with the existing theoretical power-and-interest negotiation model. Firstly, based on the findings of the case, interests should be further divided into tangible and intangible interests. The analysis of the case showed that intangible interests, such as reputation and vanity, also have certain influence on the progress of the program. Secondly, timing should be regarded as an important influential factor in program planning. Finally, "invisible" stakeholders may also affect the process and outcome of program planning.

In summary, it is necessary to further understand the power operations and interests in negotiation during adult education program planning from a Taiwanese cultural background and social context, to establish corresponding theories and research models that suit the program planning politics of adult education in Taiwan, and to which can be referred by domestic practitioners.

\section{References}

Boone, E., Safrit, D., \& Jones, J. (2002). Developing programs in adult education: A conceptual programming model (2nd ed.). Long Grove, IL: Waveland Press.

Boyle, P. (1981). Planning better programs. New York, NY: McGraw-Hill.

Cervero, R. M., \& Wilson, A. L. (1994). Planning responsibly for adult education: A guide to negotiating power and interests. San Francisco: Jossey-Bass.

Cervero, R. M., \& Wilson, A. L. (Eds.). (1996). What really matters in adult education program planning: Lessons in negotiating power and interests. In New directions for adult and continuing education (No. 69). San Francisco: Jossey-Bass.

Cervero, R. M., \& Wilson, A. L. (1998). Working the planning table: The political practice of adult education. Studies in Continuing Education, 20(1), 5-21.

Cervero, R., \& Wilson, A. (2006). Working the planning table: Negotiating democratically for adult, continuing, and workplace education. San Francisco, CA: Jossey-Bass.

Chien, H. C. (2004). Weiguan Zhengzhi yu Jiaoyu (Micropolitics and education). In T. Y. Lin (Ed.), Jiaoyu Zhengzhi Xue (Educational politics) (pp. 256-297). Taipei City: Psychology Press.

Lakoff, R. J. (1990). Talking power: The politics. Berkeley: Basic Books.

Sissel, P. A. (2001). Understanding and negotiating the political landscape of adult education. San Francisco: Jossey-Bass.

Wei, H. C. (2001). Chenren Jiaoyu Fang'an Fazhan: Lilun yu Shiji (The development of adult education programs: Theory and practice). Taipei City: Wu-Nan Culture Enterprise. 